\title{
Method of Prototyping Sensitive Dynamic Engineering Structures Supporting Automatic Information Identification Regarding a Structure's Condition
}

\author{
Tomasz Owerko \\ Department of Engineering Surveying and Civil Engineering, AGH University of Science and Technology, Cracow 30-059, Poland
}

\begin{abstract}
This publication presents an algorithm analyzing dynamic sensitive engineering structures. The measurements data which are analyzed were mostly obtained during test loading of given designs, but some examples are made also with simulated data. Data are decomposed in time and frequency domains. Hence, one is able to attain the rate of stress absorption of a given structure, in direct conjunction with the presented algorithm that is based upon the Hilbert transformation. Next, the information in stationary signals that represents a given structure before and after damage, is used to help determine the state of existing objects. Finally, the presented algorithm is capable of an in-depth analysis of new structures and monitoring existing ones, including those which are being exposed to continual use.
\end{abstract}

Key words: SHM, bridge load testing, signal processing, surveying, deformation monitoring.

\section{Introduction}

The aim of the publication is to present the algorithm used while conducting loading tests or while a structure is being used. This will allow the identification of any forecasted damages that would occur to a given structure during investigation, in turn, establishing the foundation of a reference, data-based system upon which a monitoring system could be built using the SHM (Structural Health Monitoring System). These inspections presented a basis for the assessment of such designs as to their condition in order to analyze their safety. The group of designs which must be monitored during testing while being load tested and those structures which require monitoring are bridges and buildings subject to seismic activities [1]. The monitoring of such structures and the loading tests should yield a basis for the assessment of structural integrity, hence deeming it to be operational and establish a basis for further acquisition of data as to its ongoing safety. In order to evaluate the health

Corresponding author: Tomasz Owerko, Ph.D. (eng)., research fields: engineering surveying and civil engineering. of certain structures, modern technology may be applied, which uses digital signal processing and analyzes the patterns with the use of machine learning, or analysis the statistical features of signals $[1,2]$. The analysis of the health of a structure based on the data representing the stationary signals was presented in the publications [3,4] representing an important element of the proposed solution being presented in this paper.

The subject matter of the publication is an algorithm which detects and automatically identifies structure defects, errors occurring in the construction process, especially in engineering designs; such objects which are susceptible to dynamic alteration are for instance: bridges, viaducts, pylons, masts and towers, such as free standing chimneys (single and multiple constructions). The algorithm is based upon the study of the objects while simulated loading tests were performed or while in use during testing. The technique of calculation is dependent upon the measurements which are reliant upon the measuring signals that are received by the computer as 
time-series, the spectrograms and the analysis of the stationary intervals of time series.

In the first stage, while measuring a given structure, measuring sensors which are used (including: accelerometers, interferometric radar readings of given points and GNSS (Global Navigation Satellite System) receivers) would all be placed in such a way as to:

(1) having the possibility to accurately identify the mode shapes resulting from the modal analysis of constructions;

(2) strategically find and place the sensors in areas that are prone to damage during the examinations while the structure is under load testing and also while being subjected to real users.

During the second stage, the identified values of amplitudes recorded within the outlined periods, which represent free vibrations that are contrasted with the results of the modal analysis (in the frequency domain), also in the field of values of logarithmic decrement of damping calculated on the basis of the Hilbert transformation of free vibration [5].

Next within the given periods, which represent the stationary signals, an ARMA model (linear model of autoregressive moving average) is estimated [6, 7]. On this basis, the matrix of autoregression is subjected to participial complement analysis. The DSF (damage sensitive feature) coefficients are calculated, as presented in Ref. [4], as normalized values (the first coefficients are divided by the root of sum of squares of the first three coefficients).
In the third stage, it is calculated if the distance of the given calculated coefficient, on the basis of the given time series after the crossing of a vehicle, change the coefficients of the regressive lines fitted into the previous realizations of the DSF with the use of Cook's distance. This will identify atypical behaviors of a dynamic bridge.

\section{The Essence of the Algorithm and Damping Estimation}

The signals recorded during research of load testing may be divided into three parts, in the time domain (Fig. 1):

(1) The data representing the stationary signal. This is the basis for finding the structure's features representing its condition prior to possible damage and in the parts representing the condition of the object after free vibration has expire. The second part is the basis for evaluating whether or not the force damaged the object;

(2) The data representing deflection of construction. The standard procedure may be used to calculate other parameters such as the coefficients of dynamic decrement of damping;

(3) The data representing the free vibration. Correctly filtered and standardization process allows for the calculation of an amplitude spectrum and also may determine if the design is acting in accordance with the damping based on the values of the logarithmic decrement of damping.

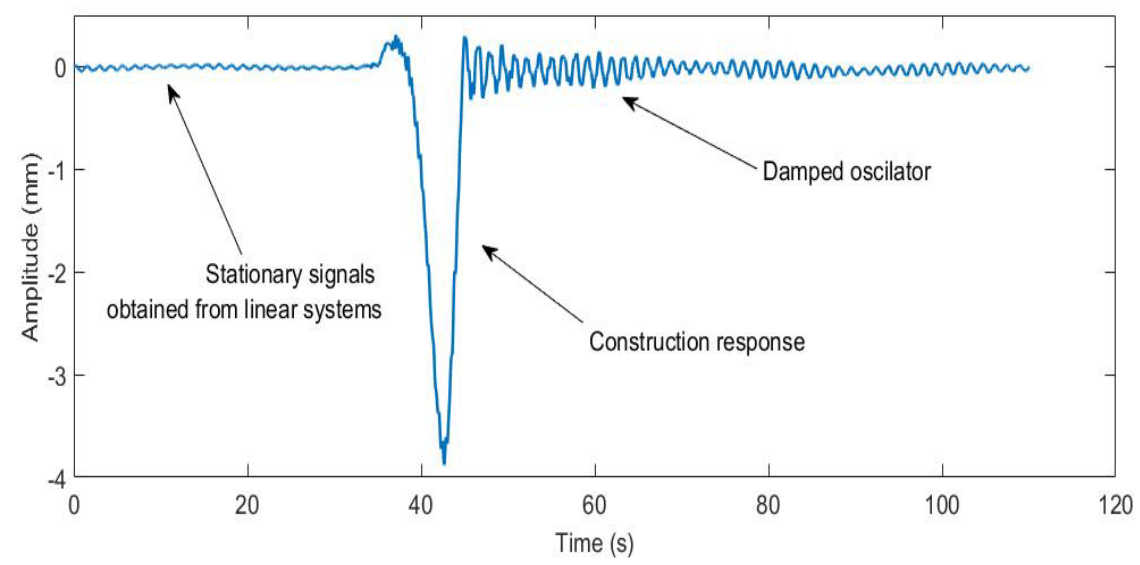

Fig. 1 The decomposition of the measurement signal. 


\subsection{Free Vibration Designs: Measuring and Analysis}

It is common practice to use free-damping data to verify FEA (Finite Element Analysis) models. Usually, data are used to obtain information about amplitude spectra (Fig. 2). In order to correctly establish an amplitude spectrum, the incoming signal must be processed with a band-pass filter supported by data as well as a pre-calculated modal analysis based on finite elements analysis method. Interval representing free vibration may be presented by Eq. (1):

$$
x(t)=A \mathrm{e}^{-B t} \cos (w t+f)
$$

where, $A$ is amplitude, $B$ is damping factor, e is Euler number, and $w, f, t$ are periods, beginning phase and time, respectively. $X$ is amplitude at time $t$.

If calculated for a given signal, the Hilbert transforms is seen in the results as an envelope of curves of a damped oscillator (Fig. 3).

Taking into account Eq. (1), the estimated parameter $B$, the damping factor, may be calculated in the following two ways:

(1) Directly from the definition by fitting exponential function into result of Hilbert transform;

(2) By fitting linear function into logarithm of Hilbert transform.

A classic logarithmic decrement of damping is calculated upon the basis of Eq. (2):

$$
D D=\ln \left(\frac{A_{n}}{A_{n+1}}\right)
$$

where, $D D$ is logarithmic decrement and $A_{n}, A_{n+1}$ are the consecutive amplitude, respectively. Directly using Eq. (2) unfortunately does not solve the problem, which is illustrated in Fig. 3. Although the band-pass filter is used, the signal, which registers the free vibration, is affected by two very similar frequencies, because the phenomenon of beat frequencies occurs in bridge structures while being tested. The proposed solution of this occurrence is superior to the classical method based upon the definition of the logarithmic decrement of damping, that in the submitted example of the estimation of damping coefficient is not hampered by the errors occurring from the number of frequency components (Fig. 4).

Such an approach allows for the estimation of the damping coefficients to be based on a robust estimation. In addition, while the estimation is being determined, the entire data acquisition of the measuring signal is being utilized, rather than a arbitrarily chosen amplitude (Fig. 4). Therefore, after determining the linear estimation, we come to

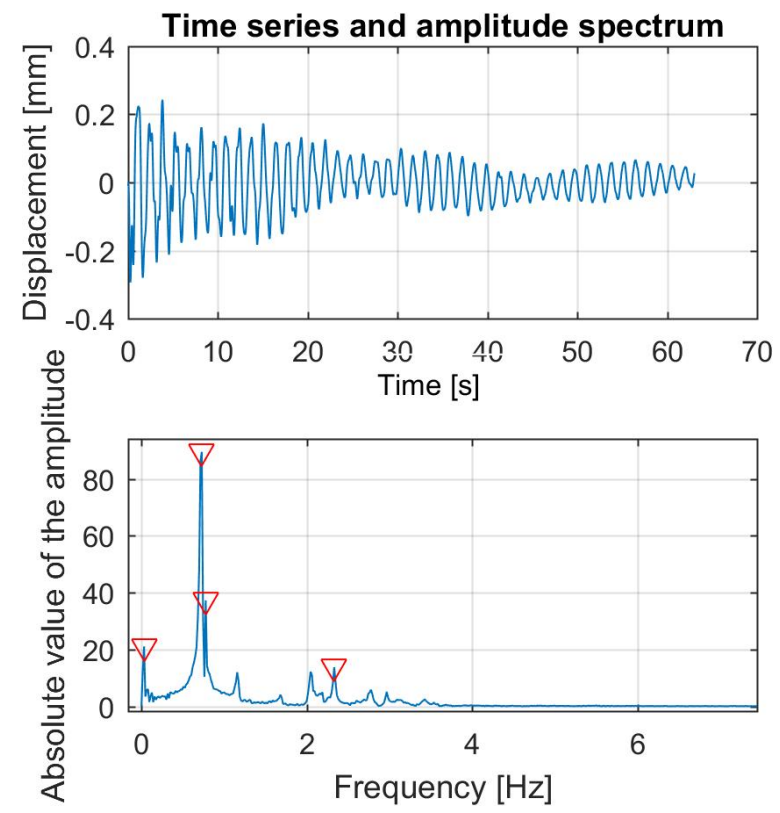

Fig. 2 Example of time series and its amplitude spectrum.

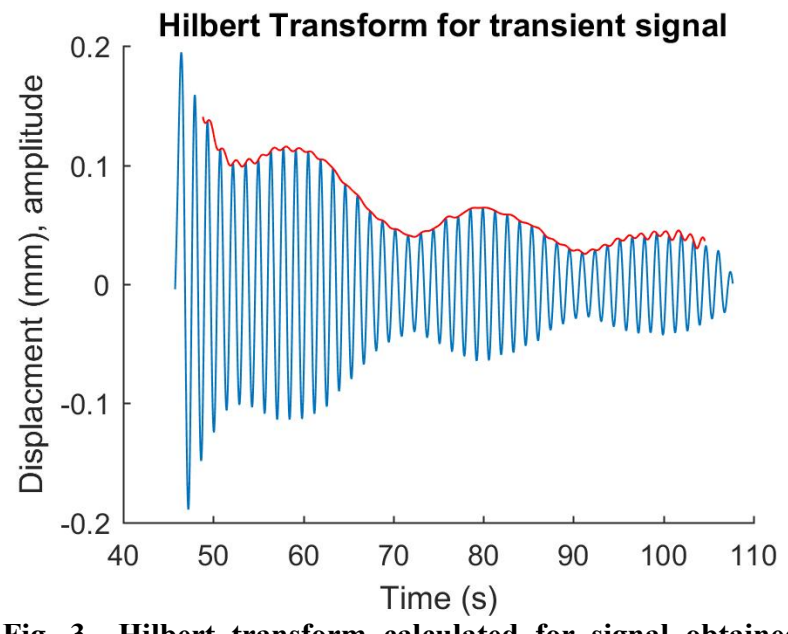

Fig. 3 Hilbert transform calculated for signal obtained from damping vibrations of bridge. Note beat frequency issue. 


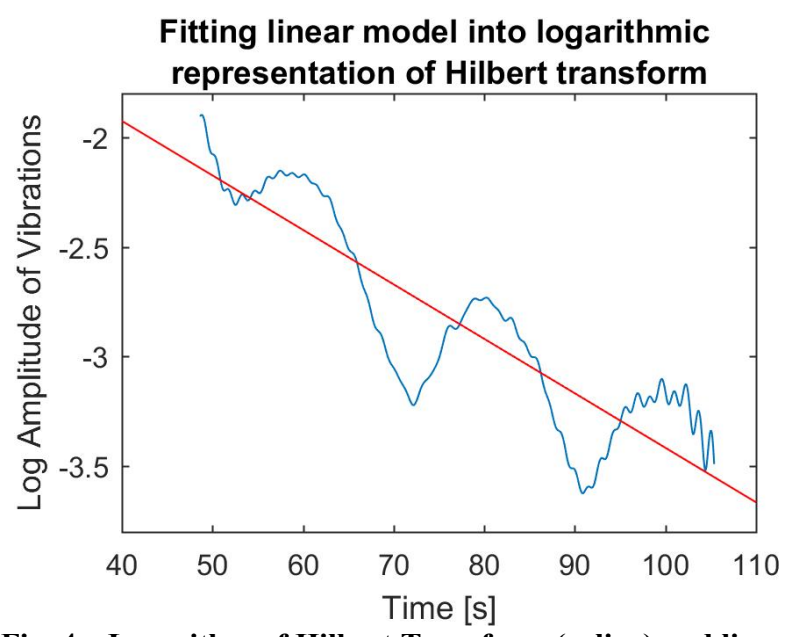

Fig. 4 Logarithm of Hilbert Transform (spline) and linear estimation (straight line).

$$
\begin{aligned}
& y=B x+C ; \\
& D D=B^{*} T
\end{aligned}
$$

where, $T$ is the period of the dampened vibration, $C$ is constant and $y$ is dependent variable.

\subsection{Structural Health Estimation and Damage Detection}

If during a load test damage to the construction occurred, it would change the statistical characteristics of the measured data. There exists a group of methods which has been developed for identification of the damage [2-4]. They are based on the congruency of the ARMA (autoregressive moving average) models into the given data. The general form is given in Eq. (4):

$$
x_{i j}(t)=\sum_{k=1}^{p} a_{k} x_{i j}(t-k)+\sum_{k=1}^{q} b_{k} e_{i j}(t-k)+e_{i j}(t)
$$

where, $x_{i j}(t)$ is the normalized measurement signal, $a_{k}$ and $b_{k}$ are the $k$ th $\mathrm{AR}$ and MA coefficients, respectively; $p$ and $q$ are the model orders of the AR and MA processes, respectively, and $e_{i j}(t)$ is the residual term. The algorithms of the group are discussed in detail in Refs. [2-4]. In particular, modified and implemented by the author, algorithm adapts the structure in Fig. 5.

\section{Structural Health Monitoring during Load Tests}

Proposed algorithmic structure can be described even more precisely. Let's assume that we would like the answer to the question if the condition of a structure before, during and after the fact it was subjected to being load tested changed at all.

Therefore, algorithm will compare in detail the intervals shown (Fig. 6) in the frames. As is shown

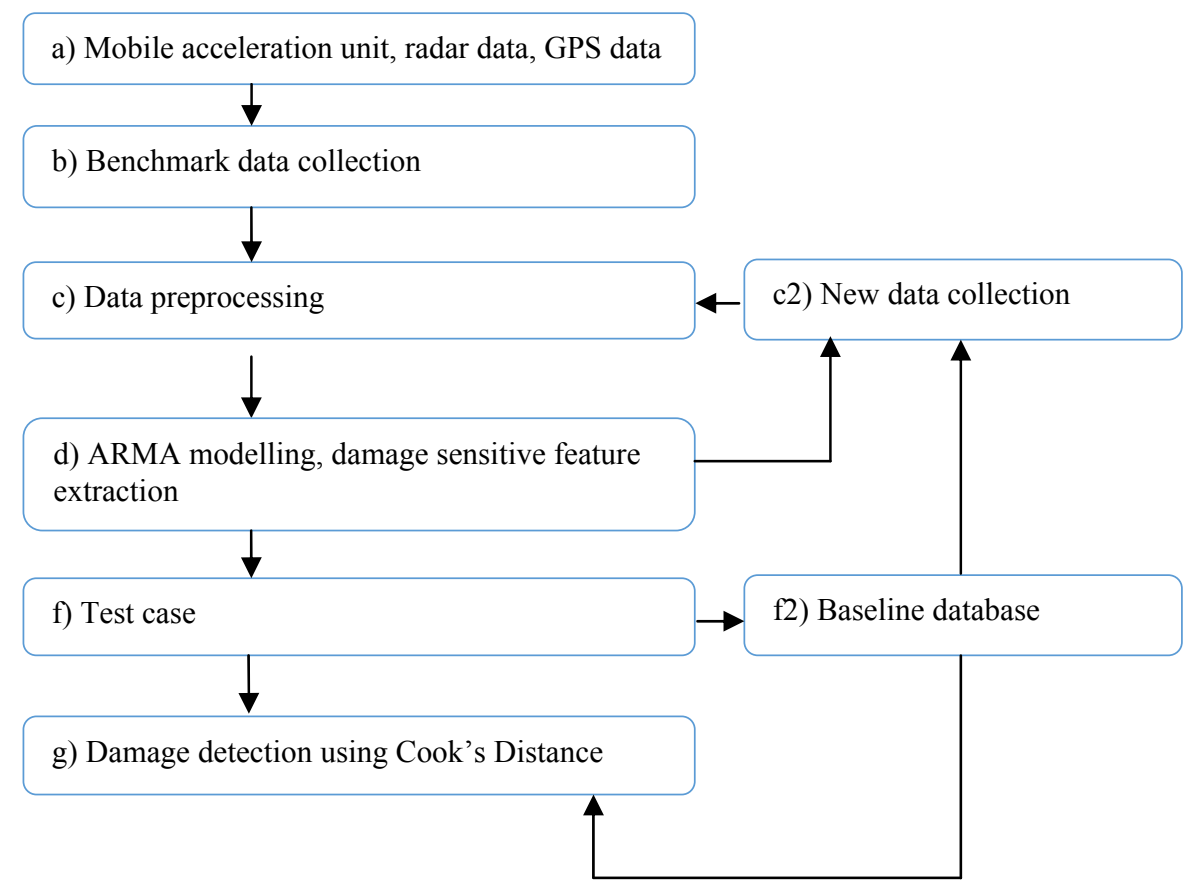

Fig. 5 Selected and implemented ARMA algorithm framework. 
(Fig. 5) in the beginning, there must be a standardized interval established. A practical way of doing this is according to Eq. (5):

$$
\bar{x}(t)=\frac{(x(t)-m)}{s t d}
$$

where, $x(t)$ is analyzed period, $m$ is the mean, while $s t d$ is standard deviation. After the standardization, the time series is entered into the model of ARMA in accordance with Eq. (4). Taking into consideration different types of engineering structures, rank of coefficients $\operatorname{AR}(p)$ and $\operatorname{MA}(q)$, in the proposed solution is subject to estimation.

Therefore, the effect of the action of the algorithm will be the results of DSF parameters (6) calculated for specific data vectors representing the engineering structure before and after potential damage (Fig. 7):

$$
D S F=\frac{a_{1}}{\sqrt{a_{1}^{2}+a_{2}^{2}+a_{3}^{2}}}
$$

where, $D S F$ is damage sensitive feature and $a_{i}$ are coefficients obtained from Eq. (4).

The classical approach to identify the damage in a given structure one must take all obtained $D S F$ coefficients prior to test (marked in Fig. 7 as circles) and use this as a basis to calculate the estimated value. The next step would be an analogical procedure for the entire interval representing the structure behavior after the excitation has been applied to construction (result is marked in Fig. 7 as plus signs).

Hence, for both groups of data, the mean values have to be estimated. Upon this basis, it may be concluded that there will be a substantial difference between the groups, using standard t-test for this aim.

This type of approach has two characteristic shortcomings:

(1) Firstly, it is crucial to take a sufficient number of samples representative of the structures behavior after force been applied to construction, in order for the statistical significance from the given test to be properly kept at accordingly high level;

(2) Limit the possibility of using the calculation

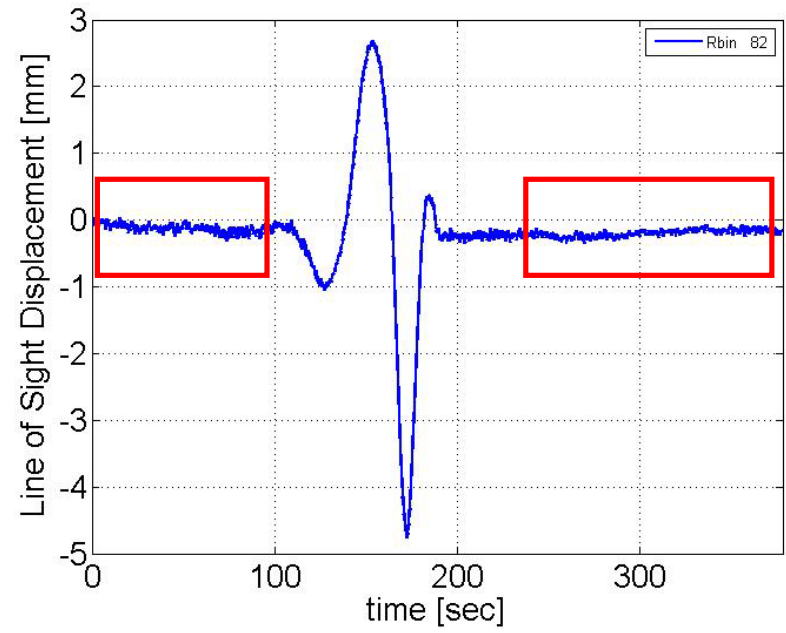

Fig. 6 Data input for time-series based damage detection based algorithms.

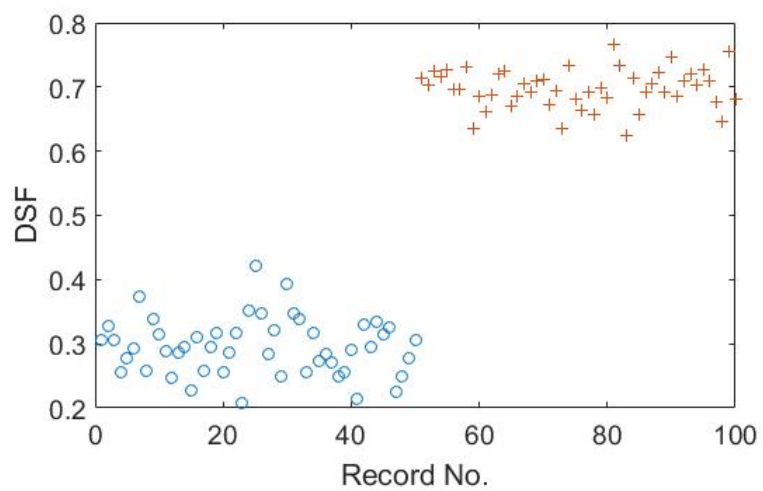

Fig. 7 DSF obtained from example data (this dataset do not represent a particular object).

techniques of bridge structures while under operation being subjected to continual use, there may not be a suitable length of time between impact of the structure to gather the proper amount of data to run a t-test determining the $D F S$ coefficients.

\section{More Efficient Way-How to Reduce the Amount of Necessary Data}

These limitations may be solved by using a different criterion than the statistical difference estimated between the two groups of data. Let's consider the groups of data from objects which after impact and damping a reading must be captured before being of next impact yet again, thus limiting the amount of information accessible-two consecutive forces are applied to structure in short 
time. In the case of such data, it is possible to calculate a limited number of DSF coefficients (in Fig. 8 identified with arrow).

Such situation may be encountered when research is being carried out in bridge structures that are in current use, especially those with a large variety of vehicles that are not standard and oversized. The question at hand is whether or not a given vehicle may be the cause of damage to a structure even during a minimal intervals between the impacts.

In order to verify whether the limited number of $D S F$ parameters that were registered are significantly different from the average realization, the formula that may be used in such a regression analysis based upon Cook's distance given in Eq. (7):

$$
D_{i}=\frac{\sum_{j=1}^{n}\left(y_{j}-y_{j(i)}\right)^{2}}{p M S E}
$$

where, $y_{j}$ is is the $j$ th fitted response value, $y_{j(i)}$ is the $j$ th fitted response value, where the fit does not include observation $i, M S E$ is the mean squared error and $p$ is the number of coefficients in the regression model. Fig. 9 presents Cook's distances calculated for our example data set. It is easy to see that all of the captured DSF coefficients for the vectors of data representing a damaged structure assigned a value above line.

The dashed line in the Fig. 9 corresponds to the recommended threshold value-three times mean Cook's distance. The plot has observations with Cook's distance values greater than the threshold value. In particular, $D S F \mathrm{~s}$ obtained for vector numbers 51, 52 and 53 have Cook's distance values that are relatively higher than the others, which exceed the threshold value. Usually, it might be necessary to find and omit these observation from your data and rebuild your model, but in our case, this information is used to answer the question if excitation (for example, passing vehicle) caused damage to the tested bridge.

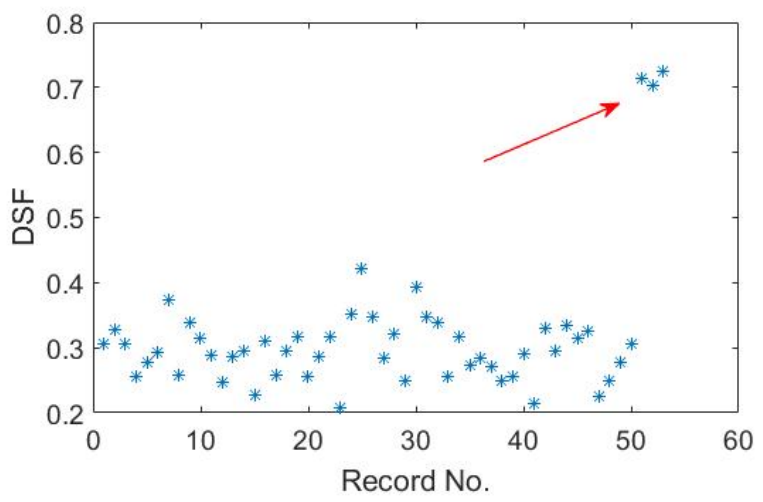

Fig. 8 DSF obtained from object where data after extraction are where limited.

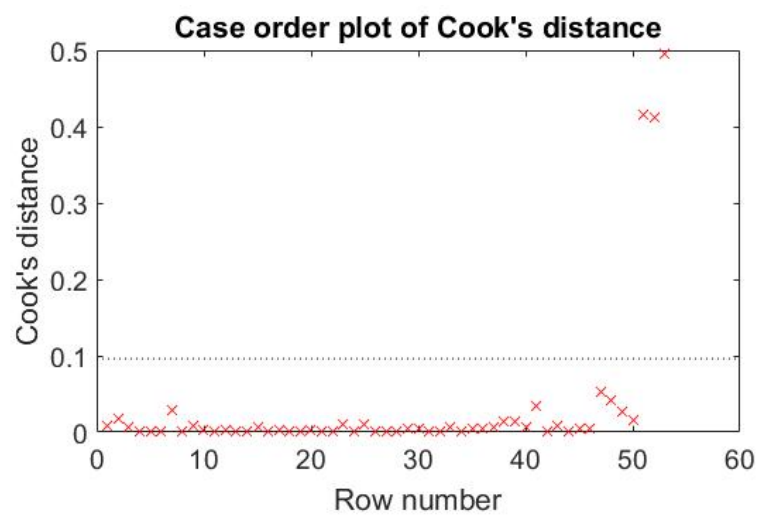

Fig. 9 Cook's distance calculated for linear model of DSF coefficients.

\section{Conclusions}

The presented algorithm comprehensively discusses the methods in which prototyping engineering structures and the detailed examination of bridge structures while load testing and actual use. The following are the basic assumptions and features:

(1) The decomposition of the recorded signal that represents the vibration of a given bridge structure into three groups in the time domain. The first group contains data before an impact and after free vibration and technically it is the group of stationary signals of a linear systems. The second group is the response of the construction (i.e., deflection of span occurred). The third group is the part of the signal which represents free vibration in a structure that is excited;

(2) The decomposition of signal in frequency spectrum, especially with band-pass filters, allows for 
the more effective spectral analysis. The band width is the results of the FEM analysis;

(3) The amplitude spectrum is comparable to the analysis made with finite elements method through the calculation of the fast Fourier transform;

(4) Construction damping of an object is represented by the logarithmical decrement. The calculation of its values is not dependent upon the implementation of the direct definition, yet on the calculation of the Hilbert transform. Furthermore, for logarithm of envelope the linear regression with robust LSF (Least Squares Fitting) fitting [8] is calculated (3). The calculated coefficients of linear estimation allow for an estimation of logarithmic decrement of damping in the entire signal, even when the structure experiences beat frequencies;

(5) The identification of potential damage to a structure as a result of impact is based in the DSF coefficients (4 and 6). The answer to the question if the damage occurred is on the basis of the Cook's distance rather than on the average values of the tests. The effect of such examinations is when in reality the conclusion may be drawn whether or not the data from the tested object indicate the damage, even in the cases when the damage occurs when tested object is in use.

It is of utmost importance that the data supporting the algorithm in the field of stationary signals were analyzed properly. Important parameters are as follows: order of ARMA model, length of data windows, test if residuals obtained are normal, impended and identically distributed. verification of construction condition has to be based on proper baseline (the same environmental conditions).

Further research is the technological implementation of machine learning which will allow for the automatic classification of the DSF coefficients.

\section{Acknowledgments}

This publication was prepared with the financial support from the statutory research fund number 11.11.150.005 of the Department of Engineering Surveying and Civil Engineering, Faculty of Mining Surveying and Environmental Engineering of AGH University of Science and Technology in Krakow.

\section{References}

[1] Farrar, C. R., and Worden, K. 2013. Structural Health Monitoring: A Machine Learning Perspective. Chichester: John Wiley \& Sons, Ltd. ISBN 978-1-119-99433-6.

[2] Wnezel, H. 2009. Health Monitoring of Bridges. Chichester: John Wiley \& Sons, Ltd. ISBN 978-0-470-03173-5.

[3] Sohn, H., Farrar, C., Hunter, H., and Worden, K. 2001. Applying the LANL Statistical Pattern Recognition Paradigm for Structural Health Monitoring to Data from a Surface-Effect Fast Patrol Boat. Technical report LA-13761-MS, Los Alamos National Laboratory, Los Alamos, New Mexico.

[4] Nair, K. K., Kiremidjian, A. S., and Law, K. H. 2006. "Time Series-Based Damage Detection and Localization Algorithm with Application to the ASCE Benchmark Structure." Journal of Sound and Vibration 291: 349-68.

[5] Shin, K., and Hammond, J. K. 2008. Fundamentals of Signal Processing for Sound and Vibration Engineers. Chichester: John Wiley \& Sons, Ltd. ISBN-13 978-0470-51188-6.

[6] Box, G. E. P., and Jenkins, G. M. 1976. Time Series Analysis: Forecasting and Control. San Francisco: Holden Day.

[7] Box, G. E. P., and Pierce, D. A. 1970. "Distribution of Residual Autocorrelation in Autoregressive Moving Avrage Time Series Models." Journal of the American Statistical Association 65: 1509-26.

[8] Neter, J., Kutner, M. H., Nachtsheim, C. J., and Wasserman, W. 1996. Applied Linear Statistical Models. 4th ed. Chicago: McGraw Hill/Irwin Series. 